\title{
Extreme thermal expansion, piezoelectricity, and other coupled field properties in composites with a negative stiffness phase
}

\author{
Y. C. Wang and R. S. Lakes ${ }^{\text {a) }}$ \\ Department of Engineering Physics, Engineering Mechanics Program, University of Wisconsin-Madison, \\ 147 Engineering Research Building, 1500 Engineering Drive, Madison, Wisconsin 53706-1687
}

(Received 17 May 2001; accepted for publication 5 September 2001)

\begin{abstract}
Particulate composites with negative stiffness inclusions in a viscoelastic matrix are shown to have higher thermal expansion than that of either constituent and exceeding conventional bounds. It is also shown theoretically that other extreme linear coupled field properties including piezoelectricity and pyroelectricity occur in layer- and fiber-type piezoelectric composites, due to negative inclusion stiffness effects. The causal mechanism is a greater deformation in and near the inclusions than the composite as a whole. A block of negative stiffness material is unstable, but negative stiffness inclusions in a composite can be stabilized by the surrounding matrix and can give rise to extreme viscoelastic effects in lumped and distributed composites. In contrast to prior proposed composites with unbounded thermal expansion, neither the assumptions of void spaces nor slip interfaces are required in the present analysis. (C) 2001 American Institute of Physics.
\end{abstract}

[DOI: $10.1063 / 1.1413947]$

\section{INTRODUCTION}

Microstructure in multiphase materials such as composites and biological tissues may be so complex that theoretical prediction of aggregate physical properties from constituent properties becomes difficult or impossible. Consequently, it is useful to develop analytical bounds upon properties in order to constrain the range of properties which may be expected. Bounds ${ }^{1,2}$ have been developed for the thermal expansion coefficient $\alpha$ of composite materials of two solid phases in terms of constituent expansion coefficients $\alpha_{1}$ and $\alpha_{2}$. The upper bound is a rule of mixtures $\alpha=\alpha_{1} V_{1}$ $+\alpha_{2}\left(1-V_{1}\right)$, in which $V_{1}$ is the volume fraction of the first phase. If the detailed microstructure of a composite is known, the exact relations of overall mechanical or coupled properties and those of each constituent can be obtained theoretically. Even if the microstructure is unknown for a particular composite, the bound equations constrain attainable behavior provided the assumptions of the bounding theorems are satisfied. Many bounding formulas are attainable, that is, they correspond exactly to a known microstructure. In deriving the bounds on thermal expansion coefficients, it was tacitly assumed that the two phases are perfectly bonded and with zero void content; moreover, that each phase is of positive stiffness with zero stored energy at equilibrium. We have shown that arbitrarily high thermal expansions can be achieved in composites with void fraction ${ }^{3}$ or in dense composites with interfaces which allow slip. ${ }^{4}$ These composites contain rib elements of composite microstructure. Each rib element is a bilayer made of two bonded layers of differing thermal expansion coefficient $\alpha$. Composites with extremal thermal expansion coefficients have also been presented based on topology optimization ${ }^{5}$ of structure

a) Author to whom correspondence should be addressed; electronic mail: lakes@engr.wisc.edu allowing void space. Inclusion of void space of appropriate shape in a composite microstructure can also give rise to unusual mechanical properties such as a negative Poisson's ratio. ${ }^{6}$ Void space is not, however, a necessary condition to achieve negative Poisson's ratio; a hierarchical laminate with dissimilar constituents also has this property. ${ }^{7}$ A common aspect is nonaffine or heterogeneous deformation. ${ }^{8}$ Negative thermal expansion coefficients are known in certain oxide systems $;{ }^{9}$ they also occur in lattice composites if the higher expansion constituent is on the convex side of each bilayer. We remark that molecular design of materials with moderate values of negative linear and volumetric thermal expansion has been conducted. ${ }^{10}$

It is the purpose of this article to explore the effects of negative stiffness composite phases in achieving extreme thermal expansion, piezoelectric, and pyroelectric coupled field properties. The possibility of extreme behavior in such composites was explored theoretically ${ }^{11}$ for viscoelastic systems. High viscoelastic damping in composite cells with negative stiffness has been demonstrated experimentally ${ }^{12}$ in compliant systems containing postbuckled tubes. High viscoelastic damping has also been observed in composites with negative stiffness particulate inclusions. ${ }^{13}$ In this article, we first demonstrate the possibility to achieve extreme thermal expansion of particulate composites via the exact relation of thermal expansion coefficients and bulk moduli. Second, we show theoretically that extreme piezoelectric constants, piezoelectric coefficients, and pyroelectric constants can also be observed in layer- and fiber-type piezoelectric composites, based on available exact or approximate relations.

\section{ANALYSIS}

Thermal expansion in composites is intimately linked with the bulk properties. The thermal expansion coefficient 
$\alpha^{c}$ of a composite is related to the bulk properties as follows, ${ }^{14,15}$ provided the composite as well as each phase is isotropic.

$$
\alpha^{c}=\alpha_{1}+\frac{\alpha_{1}-\alpha_{2}}{\frac{1}{K_{2}}-\frac{1}{K_{1}}}\left(\frac{1}{K^{c}}-\frac{1}{K_{1}}\right) .
$$

The composite thermal expansion $\alpha^{c}$ can be large if the composite bulk modulus $K^{c}$ is small. That is the case in the lattice structures considered in Refs. 3 and 4. $\alpha^{c}$ can also be large if bulk compliances of the constituents are out of proportion to their thermal expansion coefficients. Based on the statistically isotropic assumption, Eq. (1) was obtained as an exact solution by applying an eigenstress technique through the uniform fields approach, ${ }^{16}$ described as follows. The name for the uniform field was coined much later than the derivation done by Levin. First, separate the inclusions from the matrix; let both of them be loaded by a hydrostatic stress with a uniform temperature change. Second, put those inclusions back into the matrix, constrain them to satisfy the traction and displacement continuity conditions on the interfaces. Then, the expression is found for effective thermal expansion coefficients by the superposition of a hydrostatic stress in the opposite direction on the outer boundary of the matrix to cancel the one added artificially in the first step.

As for the bulk modulus as input to Eq. (1), consider the Hashin-Shtrikman ${ }^{17}$ (1963) formulas. The lower bounds for the elastic shear modulus $G_{L}$ and bulk modulus $K_{L}$ of a composite are:

$$
\begin{aligned}
& G_{L}=G_{2}+\frac{V_{1}}{\frac{1}{G_{1}-G_{2}}+\frac{6\left(K_{2}+2 G_{2}\right) V_{2}}{5\left(3 K_{2}+4 G_{2}\right) G_{2}},} \\
& K_{L}=K_{2}+\frac{V_{1}\left(K_{1}-K_{2}\right)\left(3 K_{2}+4 G_{2}\right)}{\left(3 K_{2}+4 G_{2}\right)+3\left(K_{1}-K_{2}\right) V_{2}},
\end{aligned}
$$

in which $K_{1}$ and $K_{2}, G_{1}$ and $G_{2}$, and $V_{1}$ and $V_{2}$ are the bulk modulus, shear modulus, and volume fraction of phases 1 , and 2, respectively. If $G_{1}>G_{2}$, then $G_{L}$ represents the lower bound on the shear modulus. Interchanging the subscripts 1 and 2 results in the upper bound $G_{U}$ for the shear modulus. The bounds for isotropic composites are attainable. The Hashin-Shtrikman formula for the bulk modulus, Eq. (3), is attained exactly by a coated sphere morphology. The "lower" composite corresponds to the case of stiff spheres coated with a compliant layer. The shear modulus of the coated sphere morphology approximates the corresponding Hashin-Shtrikman formula, Eq. (2). Exact attainment of Eq. (2), however, is possible via a laminate morphology ${ }^{18}$ as shown by Milton.

We now allow one phase to have negative stiffness. Negative stiffness entails a reversal of the usual directional relationship between force and displacement in deformed objects. It does not violate any physical law, but an isolated object with negative stiffness is unstable. Composite materials with negative stiffness inclusions can be stable provided the inclusion stiffness is not excessively negative. In elastic composites, the elastic moduli can become singular if the

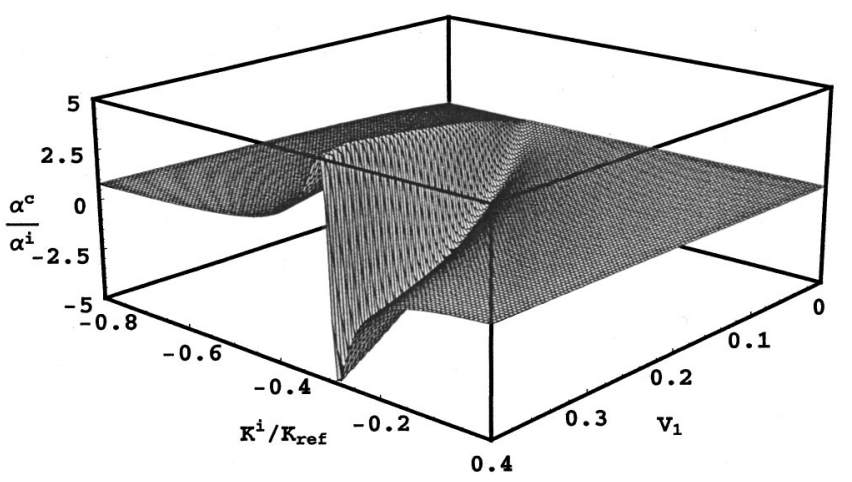

FIG. 1. The thermal expansion coefficient (real part of $\alpha_{c}$ ) of a HashinShtrikman composite assuming a matrix phase of mechanical damping $\tan \delta$ $=0.05$ in the bulk modulus with negative stiffness inclusions, as a function of inclusion stiffness and volume fraction. Thermal expansion coefficients of inclusion and matrix differ by a factor of two. The composite thermal expansion coefficient is normalized to that of phase 1 .

phases of positive and negative stiffness are properly balanced. In viscoelastic composites ${ }^{11}$ with a negative stiffness phase, an anomaly in stiffness is predicted, as well as a peak in the mechanical damping. In that analysis, we applied the elastic-viscoelastic correspondence to the exact relations (1) and (3) to obtain corresponding relations for viscoelastic media. These formulas no longer represent bounds, however they are exact solutions for particular microstructures.

If the bulk modulus of one phase is allowed to become negative, the thermal expansion coefficient can greatly exceed that of either phase, as shown in Fig. 1, which is based on Eq. (1), and the lower bound formula for effective bulk modulus, Eq. (3). As inclusion stiffness is reduced, the composite thermal expansion attains its maximum magnitude first, then the composite bulk modulus attains a minimum. The enhancement of thermal expansion becomes singular as the mechanical damping of the phases tends to zero, as shown in Fig. 2. The singularity in thermal expansion occurs as the composite bulk modulus tends to zero. Therefore, the present composites share with prior ones ${ }^{3-5}$ the characteristic that extreme expansion is associated with bulk compliance. We remark that singular thermal expansion can be achieved without the composite bulk modulus passing through negative regions in which stabilization might be required. Specifically, in Fig. 2, as the inclusion bulk modulus is tuned through progressively more negative values, the composite thermal expansion becomes singular before the composite bulk modulus.

As for the piezoelectric coupling problems, Skinner, Newnham, and Cross ${ }^{19}$ proposed a set of rule-of-mixtures type equations to approximately estimate the effective piezoelectric coefficients, as follows.

$$
\begin{aligned}
& \left(d_{33}^{c}\right)_{\text {series }}=\frac{V_{1} d_{33}^{i} \varepsilon_{33}^{m}+\left(1-V_{1}\right) d_{33}^{m} \varepsilon_{33}^{i}}{V_{1} \varepsilon_{33}^{m}+\left(1-V_{1}\right) \varepsilon_{33}^{i}}, \\
& \left(d_{33}^{c}\right)_{\text {parallel }}=\frac{V_{1} d_{33}^{i} S_{33}^{m}+\left(1-V_{1}\right) d_{33}^{m} S_{33}^{i}}{V_{1} S_{33}^{m}+\left(1-V_{1}\right) S_{33}^{i}}, \\
& d_{31}^{c}=V_{1} d_{31}^{i}+\left(1-V_{1}\right) d_{31}^{m},
\end{aligned}
$$




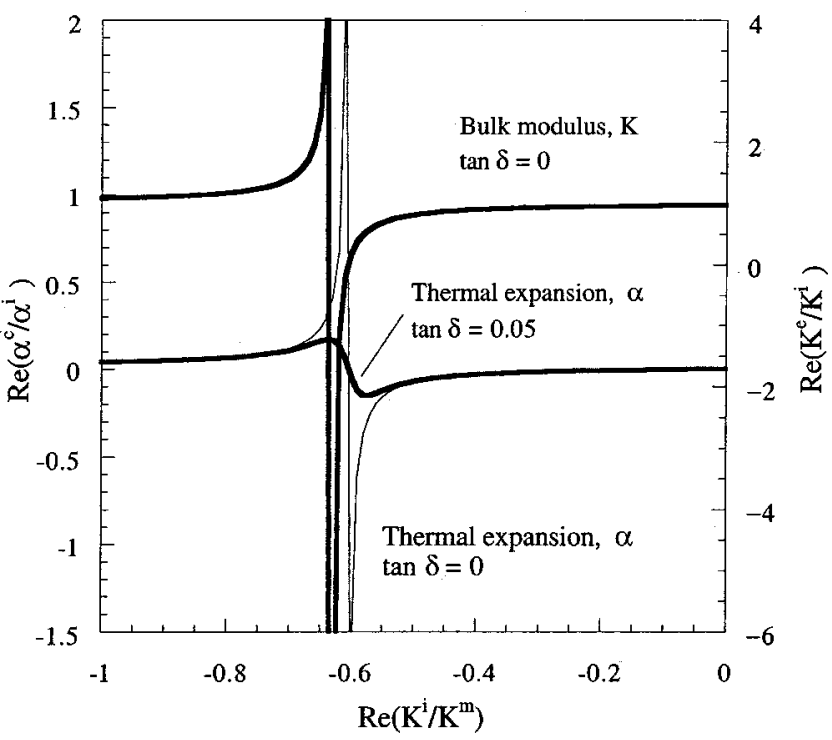

FIG. 2. Thermal expansion properties of a Hashin-Shtrikman composite assuming a inclusion phase of mechanical damping $\tan \delta=0.05$ or 0 and negative stiffness. Thermal expansion is normalized to that of phase 1 . The volume fraction of the inclusion is 0.01 .

where the symbols $d_{j k}, \varepsilon_{j k}$, and $S_{j k}$ are piezoelectric coefficients, permittivity constants, and elastic compliances, the inverse of the elastic modulus $\left(C_{j k}\right)$. The indexes $j$ and $k$ of second rank tensor properties $\varepsilon_{j k}$ are from 1 to 3, denoting the axes of the coordinate system. The subscripts, series and parallel, represent the type of connectivity, which corresponds to the Reuss and Voigt composites, respectively, in geometry. In the series case, 3-axis represents the axis perpendicular to the layers; the reverse applies in the parallel case. The superscripts $i, m$, and $c$ represent the inclusion, matrix, and composite. The relationship between piezoelectric constants $\left(e_{j k}\right)$ and piezoelectric coefficients $\left(d_{j k}\right)$ is $d_{j k}=e_{j q} S_{q k}$. The usual contracted index notation is used for the higher order tensorial properties, such as elastic moduli $\left(C_{i j k l}\right)$, elastic compliances $\left(S_{i j k l}\right)$, and piezoelectric properties $\left(e_{i j k}\right.$ or $\left.d_{i j k}\right)$ in which only the last two indexes are contracted, as used by Nye. ${ }^{20}$ The contraction means indexes 11 become 1, 22 become 2, 33 become 3, 23 become 4, 13 become 5,12 become 6 . Each of the field quantities is measured when all others are fixed. A more rigorous derivation, based on the linear coupled field theory and uniform field technique, for this sort of two-phase layer-type piezoelectric composites, consisting of transversely isotropic phases, is given by Benveniste and Dvorak. ${ }^{21}$ The 17 independent material parameters for the overall orthotropic piezoelectric composites are obtained exactly.

To simplify our theoretical analysis of this coupled field problem, we assume each of the piezoelectric transducer (PZT) inclusion layers or rods is mechanically isotropic. This assumption is justified since the degree of mechanical anisotropy of certain PZT materials, for example PZT-5A (Ref. 22) is not very pronounced. Moreover, most piezoelectric ceramics are rather stiff, therefore typical matrix materials can be expected to be much more compliant than piezoelectric inclusions. In the vicinity of a phase transformation, a single domain can go from positive to negative stiffness even if the
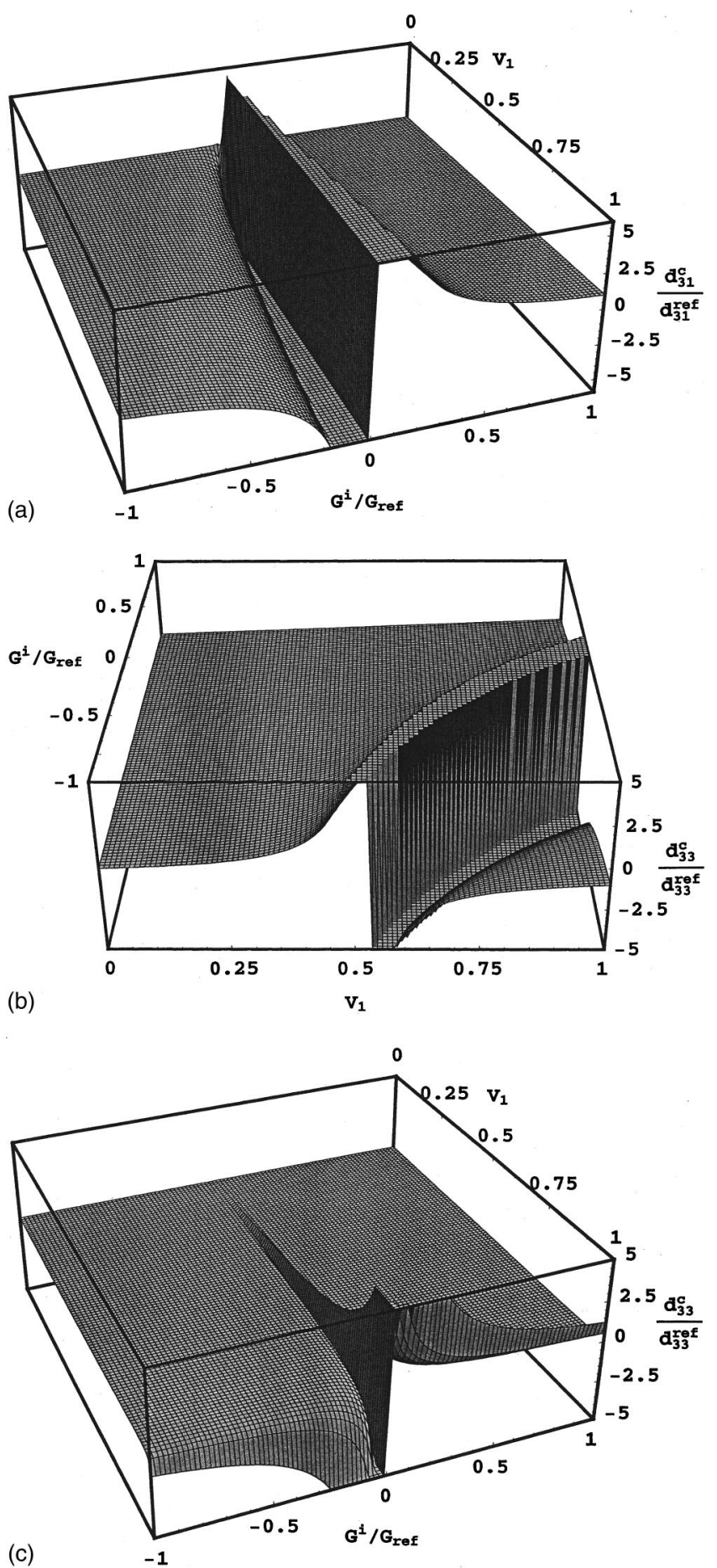

(c)

FIG. 3. (a) Normalized piezoelectric coefficient $d_{33}$, in series, (b) normalized piezoelectric coefficient $d_{33}$, in parallel, and (c) normalized piezoelectric coefficient $d_{31}$ of a layer-type piezoelectric composite versus inclusion volume fraction $\left(V_{1}\right)$ and normalized inclusion shear modulus $\left(G^{i} / G_{\mathrm{ref}}\right)$, where $G_{\text {ref }}=25 \mathrm{GPa}$. Calculated by Eqs. (4) $-(6)$.

parent material is stiff away from the transition. Therefore, to delineate phenomena, we allow the shear modulus of the inclusion to become negative. To aid the numerical calculation of the overall piezoelectric properties, we assume the electrical and coupled properties of the inclusion to be the same as those of PZT-5A material, ${ }^{24}$ which are $-5.4,15.8$, and $12.3\left(\mathrm{C} / \mathrm{m}^{2}\right)$ for $e_{31}, e_{33}$, and $e_{15}$, as well as $916 \varepsilon_{0}$, 

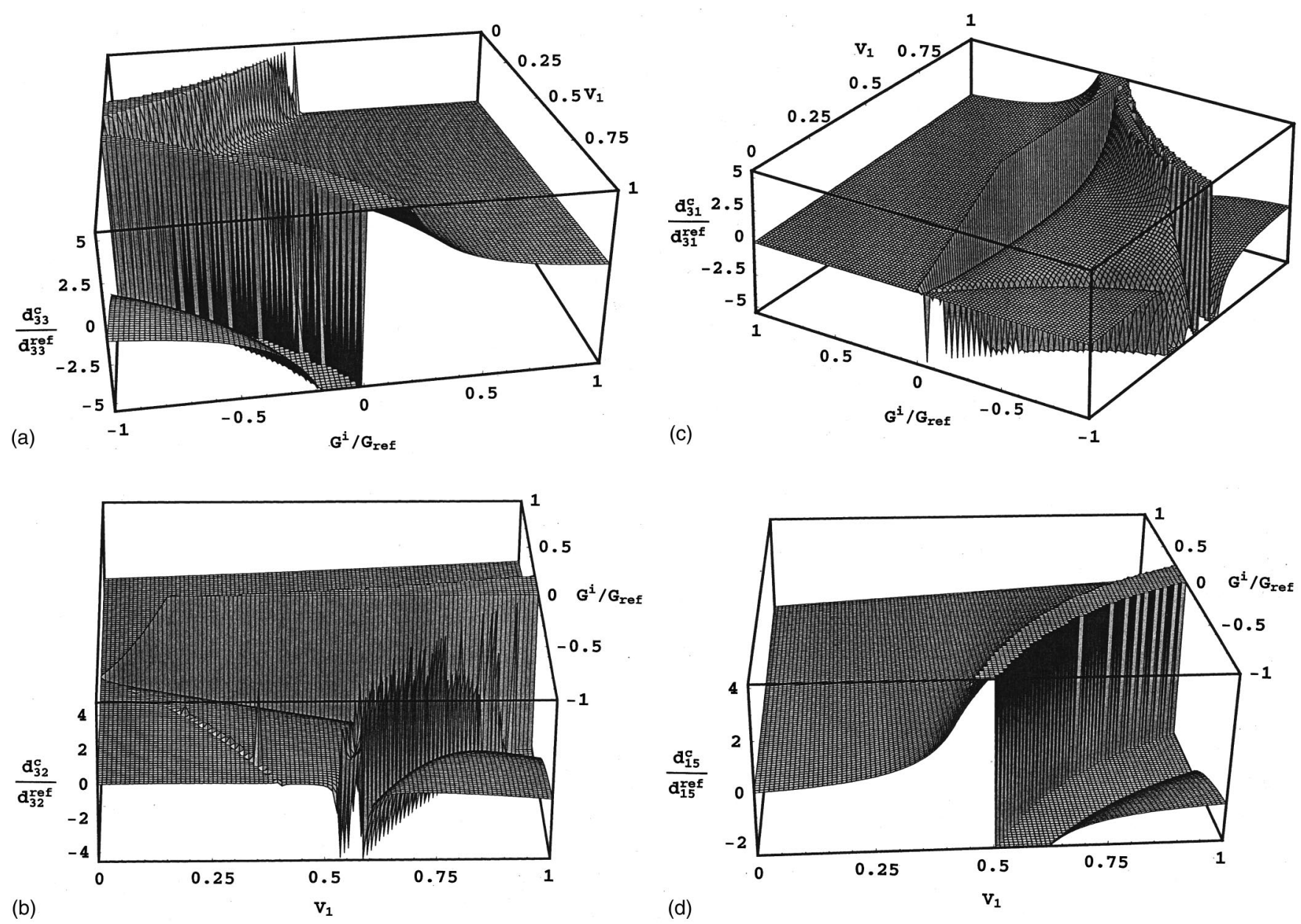

FIG. 4. (a) Normalized piezoelectric coefficient $d_{33}$, (b) normalized piezoelectric coefficient $d_{32}$, (c) normalized piezoelectric coefficient $d_{31}$, and (d) normalized piezoelectric coefficient $d_{15}$ of a layer-type piezoelectric composite versus inclusion volume fraction $\left(V_{1}\right)$ and normalized inclusion shear modulus $\left(G^{i} / G_{\text {ref }}\right)$, where $G_{\text {ref }}=25 \mathrm{GPa}$. Calculated by the equations, which are exact, in Ref. 21.

$830 \varepsilon_{0}$ for $\varepsilon_{11}$ and $\varepsilon_{33}$, where $\varepsilon_{0}$ represents the permittivity in a vacuum. For the isotropic matrix, there are no coupled electrical properties, but we assume its permittivity to be $4.43 \varepsilon_{0}$. Figure 3 shows the existence of singularities, predicted by Eqs. (4)-(6), in this type of composites for their piezoelectric coefficients when their piezoelectric phases undergo a negative stiffness transition. Based on those equations obtained by Benveniste and Dvorak, the singularities also can be observed in the graphical results, Fig. 4, for the overall piezoelectric coefficients $d_{33}, d_{32}, d_{31}$, and $d_{15}$, in which the index 3 indicates the axis perpendicular to the layers.

In the piezoelectric composites with 1-3 connectivity, contrasted with the aforementioned piezoelectric composites, which are catalogued as those with 2-2 connectivity, the piezoelectric phase is continuously self-connected in one dimension and the matrix is connected in three dimensions. In other words, they are fiber-type or transversely isotropic composites. Throughout our analysis for fiber composites, the 3 direction of the composite is along the direction in which the fibers are aligned and poled. Benveniste ${ }^{23}$ pointed out, among the ten independent material constants for the overall properties, nine of them can be derived exactly as simple expressions in the model of composite cylinder as- semblage. Because of the independence of electric field, the relation for effective plane strain bulk modulus, Eq. (7) is exact, based on the same argument used for the effective bulk modulus in the Hashin-Shtrikman bounds.

$$
k^{c}=k^{m}+\frac{V_{1}}{\frac{1}{k^{i}-k^{m}}+\frac{1-V_{1}}{k+G_{T}^{m}}},
$$

where $k$ is the plane strain bulk modulus, and $G_{T}$ is the transverse shear modulus, differing from $G_{A}$, which is denoted as the longitudinal shear modulus. Following the universal connection for piezoelectric fiber-type composites, found by Schulgasser, ${ }^{24}$ we can obtain the following exact relations.

$$
\begin{aligned}
C_{13}^{c}= & \frac{\left(k^{m}-k^{c}\right) C_{13}^{i}-\left(k^{i}-k^{c}\right) C_{13}^{m}}{k^{m}-k^{i}}, \\
C_{33}^{c}= & V_{1} C_{33}^{i}+V_{2} C_{33}^{m} \\
& -\frac{\left(C_{13}^{m}-C_{13}^{c}\right)\left(V_{1} C_{13}^{i}+V_{2} C_{13}^{m}-C_{13}^{c}\right)}{k^{m}-k^{c}},
\end{aligned}
$$



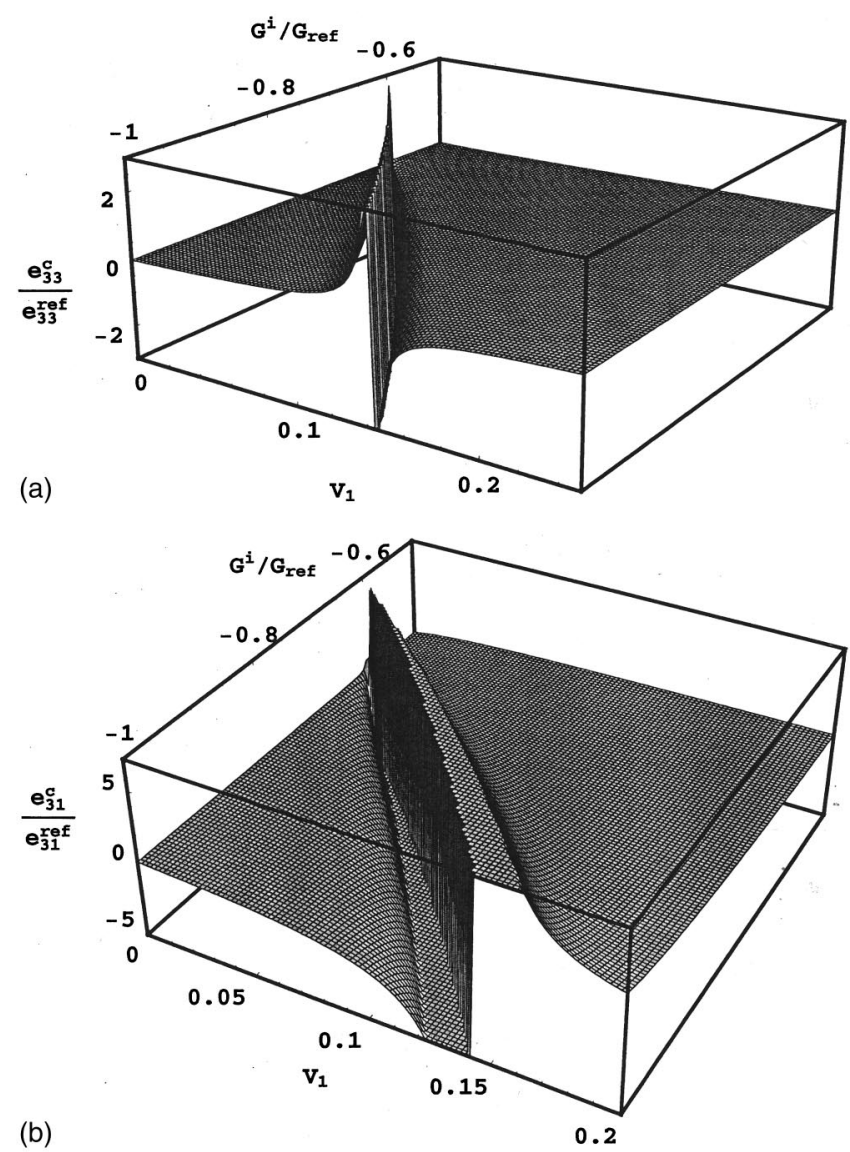

(b)

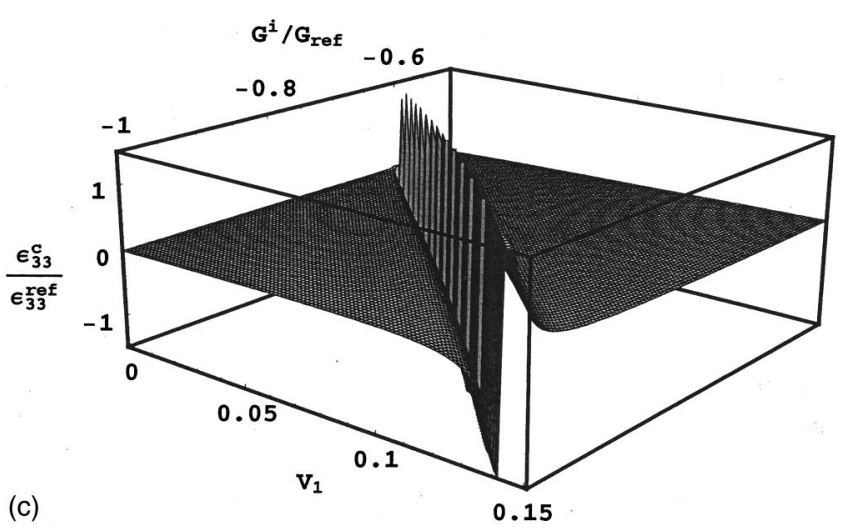

FIG. 5. (a) Normalized piezoelectric coefficient $e_{33}$, (b) normalized piezoelectric coefficient $e_{31}$, and (c) normalized permittivity constant $\varepsilon_{33}$ of a 1-3 fiber-type piezoelectric composite versus inclusion volume fraction $\left(V_{1}\right)$ and normalized inclusion shear modulus $\left(G^{i} / G_{\text {ref }}\right)$, where $G_{\text {ref }}$ $=25 \mathrm{GPa}$. Calculated by Eqs. (10)-(12), exact relations.

$$
\begin{aligned}
e_{33}^{c}= & V_{1} e_{33}^{i}+\left(1-V_{1}\right) e_{33}^{m} \\
& -\frac{\left(e_{31}^{m}-e_{31}^{c}\right)\left(V_{1} C_{13}^{i}+\left(1-V_{1}\right) C_{13}^{m}-C_{13}^{c}\right)}{k^{m}-k^{c}}, \\
e_{31}^{c}= & \frac{e_{31}^{i}\left(k^{m}-k^{c}\right)-e_{31}^{m}\left(k^{i}-k^{c}\right)}{k^{m}-k^{i}}, \\
\varepsilon_{33}^{c} & =V_{1} \varepsilon_{33}^{i}+V_{2} \varepsilon_{33}^{m}-\frac{\left(e_{31}^{m}-e_{31}^{c}\right)\left(e_{31}^{c}-V_{1} e_{31}^{i}-V_{2} e_{31}^{m}\right)}{k^{m}-k^{c}},
\end{aligned}
$$

The rest of the exact relations for $e_{15}, G_{A}$, and $\varepsilon_{11}$, demonstrated by Milgrom and Shtrikman, ${ }^{25}$ are presented as follows.

$$
\begin{aligned}
\mathbf{L}^{c}= & \mathbf{L}^{m}\left[\left(1-V_{1}\right) \mathbf{L}^{i}+\left(1+V_{1}\right) \mathbf{L}^{m}\right]^{-1}\left[\left(1+V_{1}\right) \mathbf{L}^{i}\right. \\
& \left.+\left(1-V_{1}\right) \mathbf{L}^{m}\right],
\end{aligned}
$$

where

$$
\mathbf{L}^{r}=\left[\begin{array}{cc}
G_{L} & e_{15} \\
e_{15} & -\epsilon_{11}
\end{array}\right]^{r}, \quad r=i, m .
$$

Figure 5 shows the singularities in piezoelectric constants, $e_{31}$ and $e_{33}$, and permittivity $\varepsilon_{33}$, predicted by the exact solutions, Eqs. (10)-(12). For the transversely isotropic materials modeled by composite cylinder assemblage, the effective transverse shear modulus can not be determined exactly. ${ }^{23}$ However, deriving this effective property is a purely mechanical problem, since there is no coupling with electric field. Therefore, one can use, for example, generalized self-consistent method ${ }^{26}$ to calculate it approximately. Alternatively, by the multiple-scattering technique, all of the ten effective material properties can be computed by Eqs. (14)-(23), plus the pyroelectric constant $p_{3}$ by Eq. (24), ${ }^{27,28}$ presented next, in the sense of a first order approximation. The importance of these equations is that they represent the overall properties of the two-phase transversely isotropic piezoelectric composites, whose microstructure is comprised of infinitely long fibers. Mathematically, they are equivalent to the results of the self-consistent effective-medium theory, ${ }^{29}$ after the $V_{1}$ square terms are ignored in the effective elastic moduli.

$$
\begin{aligned}
& k^{c}=\frac{k_{2}\left(k_{1}+m_{2}\right)+V_{1} m_{2}\left(k_{1}-k_{2}\right)}{k_{1}+m_{2}-V_{1}\left(k_{1}-k_{2}\right)} \\
& m^{c}=m_{2} \frac{k_{2}\left(m_{1}+m_{2}\right)+2 m_{1} m_{2}+V_{1} k_{2}\left(m_{1}-m_{2}\right)}{k_{2}\left(m_{1}+m_{2}\right)+2 m_{1} m_{2}-V_{1}\left(m_{1}-m_{2}\right)\left(k_{2}+2 m_{2}\right)}
\end{aligned}
$$

$l^{c}=\frac{C_{12}^{m}\left(k_{1}-k^{c}\right)+C_{13}^{i}\left(k^{c}-k_{2}\right)}{k_{1}-k_{2}}$

$n^{c}=C_{11}^{m}+V_{1}\left(C_{33}^{i}-C_{11}^{m}\right)-2 V_{1} \frac{\left(C_{13}^{i}-C_{12}^{m}\right)^{2}\left(k_{2}-k^{c}\right)}{\left(k_{1}+m_{2}\right)\left(k_{1}-k_{2}\right)}$

$p^{c}=\frac{m_{2}\left(m_{2}+2 C_{55}^{i}\right)+V_{1} m_{2}\left(2 C_{55}^{i}-m_{2}\right)}{2\left(m_{2}+2 C_{55}^{i}\right)+2 V_{1}\left(m_{2}-2 C_{55}^{i}\right)}$

$e_{31}^{c}=V_{1} e_{31}^{i} \frac{k^{c}+m_{2}}{k_{1}+m_{2}}$

$e_{33}^{c}=V_{1} e_{31}^{i}-2 V_{1} e_{31}^{i} \frac{C_{13}^{i}-l^{c}}{k_{1}+m_{2}}$

$e_{15}^{c}=\frac{V_{1} e_{15}^{i} \varepsilon_{2}\left(m_{2}+2 p^{c}\right)}{\left[\left(1+V_{1}\right) \varepsilon_{2}+\left(1-V_{1}\right) \varepsilon_{11}^{i}\right]\left(m_{2}+2 C_{55}^{i}\right)}$ 
$\varepsilon_{11}^{c}=\varepsilon_{2}$

$$
\times \frac{V_{2} \varepsilon_{2}+\left(1+V_{1}\right) \varepsilon_{11}^{i}-2 V_{1}\left(e_{15}^{c}-e_{15}^{i}\right) e_{15}^{i} /\left(m_{2}+2 C_{55}^{i}\right)}{\left(1+V_{1}\right) \varepsilon_{2}+V_{2} \varepsilon_{11}^{i}}
$$

$\varepsilon_{33}^{c}=\varepsilon_{2}+V_{1}\left(\varepsilon_{33}^{i}-\varepsilon_{2}\right)-2 V_{1} e_{31}^{i} \frac{e_{31}^{c}-e_{31}^{i}}{k_{1}+m_{2}}$

$p_{3}^{c}=V_{1}\left(p_{3}^{i}-\frac{V_{2}}{V_{1}} \frac{2 \beta_{2} e_{31}^{c}}{k_{2}+m_{2}}+\frac{2 \beta_{11}^{i}\left(e_{31}^{i}-e_{31}^{c}\right)}{k_{2}+m_{2}}\right)$,

in which $k / 2, m / 2, l, n, p$ are Hill's ${ }^{30}$ elastic moduli under a constant electric and temperature field. The single subscripts represent the inclusion, as 1 , and the matrix, as 2 . In contrast, the double subscripts represent second order tensors. The symbols $p_{3}, \beta$ represent pyroelectricity and thermal stress coefficient. This pyroelectricity is the variation of electric displacement along the 3 -axis due to a unit change of temperature. The relation of $\beta$ to the thermal expansion coefficients is $\beta=C \alpha$.

To investigate the anomaly in the pyroelectric constants, we adopt the CdS-epoxy piezoelectric composite, ${ }^{28}$ for

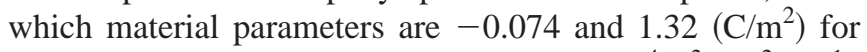
$e_{31}$ and $e_{33}, 1.17 \varepsilon_{0}$ for $\varepsilon_{33}$, and $-0.04\left(10^{-4} \mathrm{C}^{2}-\mathrm{m}^{-2}-\mathrm{K}^{-1}\right)$ for $p_{3}$. Also, the degree of mechanical anisotropy of the inclusion is low. The thermal properties of the inclusion and matrix are 6.5 and $4\left(10^{-6} \mathrm{~K}^{-1}\right)$ for $\alpha_{11}$ and $\alpha_{33}$ of the inclusion, and $60\left(10^{-6} \mathrm{~K}^{-1}\right)$ for the thermally isotropic matrix. It is noteworthy that, in all of our analysis, the absolute values of these material properties are not important because only the normalized values are of interest to demonstrate the anomalies. The standard normalization method used here is to divide each of the overall composite properties by that of phase 1 , correspondingly, except for the shear modulus of the inclusion, which is normalized to that of phase 2, and the overall piezoelectric coefficients, which are normalized to those evaluated at $V_{1}=1$ and $G^{i} / G_{\text {ref }}=1$. In our calculation, shear modulus is tuned through negative values while maintaining coupled field constants unchanged. Single domains may approximate such behavior but polydomain blocks would not. Here, since we are considering linear systems, the influence of nonlinear behavior of piezoelectric materials, such as ferroelectricity, ${ }^{31}$ upon the homogenization of composites is neglected. Figure 6 shows the singularity in the composite piezoelectric constants $e_{31}$ and $e_{33}$, as inclusion stiffness becomes negative. Figures 7 and 8 show that singularities also occur in the composite piezoelectric coefficients, $d_{33}, d_{32}, d_{31}$, and $d_{15}$, and the permittivity $\varepsilon_{33}$, as the inclusion stiffness becomes negative. Figure 9 shows the influence of inclusion negative stiffness on the composite pyroelectric property. As with the other coupled fields, singularities occur for proper values of inclusion stiffness. As with the viscoelastic case, ${ }^{11}$ the singularities in the coupled fields become peaks of finite magnitude when material damping properties of either phase are considered.
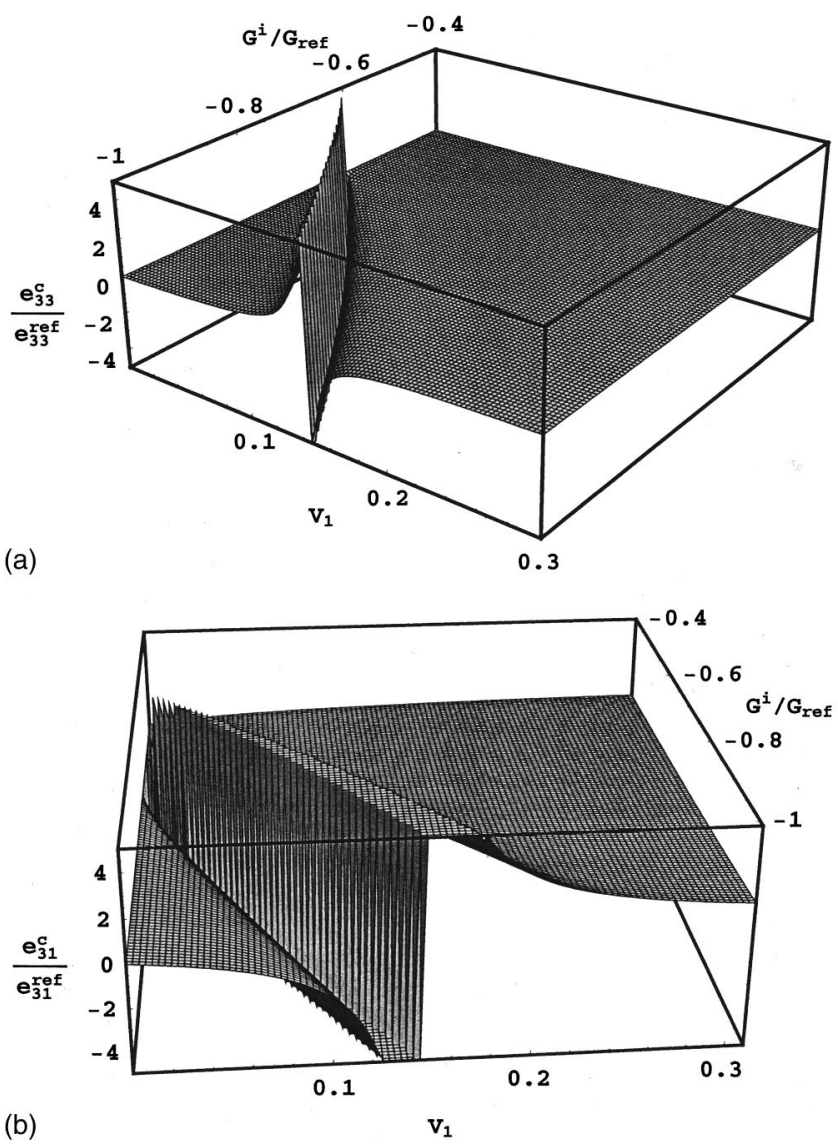

FIG. 6. (a) Normalized piezoelectric constant $e_{33}$ and (b) normalized piezoelectric constant $e_{31}$ of a 1-3 fiber-type piezoelectric composite versus inclusion volume fraction $\left(V_{1}\right)$ and normalized inclusion shear modulus $\left(G^{i} / G_{\text {ref }}\right)$, where $G_{\text {ref }}=25 \mathrm{GPa}$. Calculated by Eqs. (19) and (20).

\section{DISCUSSION}

Although a giant magnitude of thermal expansion coefficients can be also achieved by introducing the concepts of void phase or slip interfaces, ${ }^{3,4}$ the present approach not only largely increases the expansion of materials in magnitude, but also improves the properties of stiffness and mechanical damping simultaneously. ${ }^{11-13}$ In addition, the layer type of this kind of composites makes the actuator application, such as cantilever beam bimorph actuators, ${ }^{32}$ more feasible at a designated temperature, without microdevice fabrication. The achievement of enhanced composite stiffness by negative stiffness inclusions, while supported experimentally, ${ }^{13}$ raises questions of stability. ${ }^{33}$ For thermoelasticity or piezoelectricity, the sign is usually immaterial in transducer or sensor applications. Consequently, we expect it will be easier to achieve overall stability in composites intended for extreme thermoelastic or piezoelectric coupling. Tuning of negative stiffness can be achieved by control of temperature through a phase transition ${ }^{13}$ or by controlling prestrain upon a postbuckled system. ${ }^{12}$ The negative stiffness approach is distinct from the use of structural resonance ${ }^{34}$ to achieve high dielectric properties, since there are no inertial terms in the continuum elasticity equations used in the present work.

Piezoelectric composite materials ${ }^{35}$ are of interest since the figure of merit for a variety of sensor and actuators can 

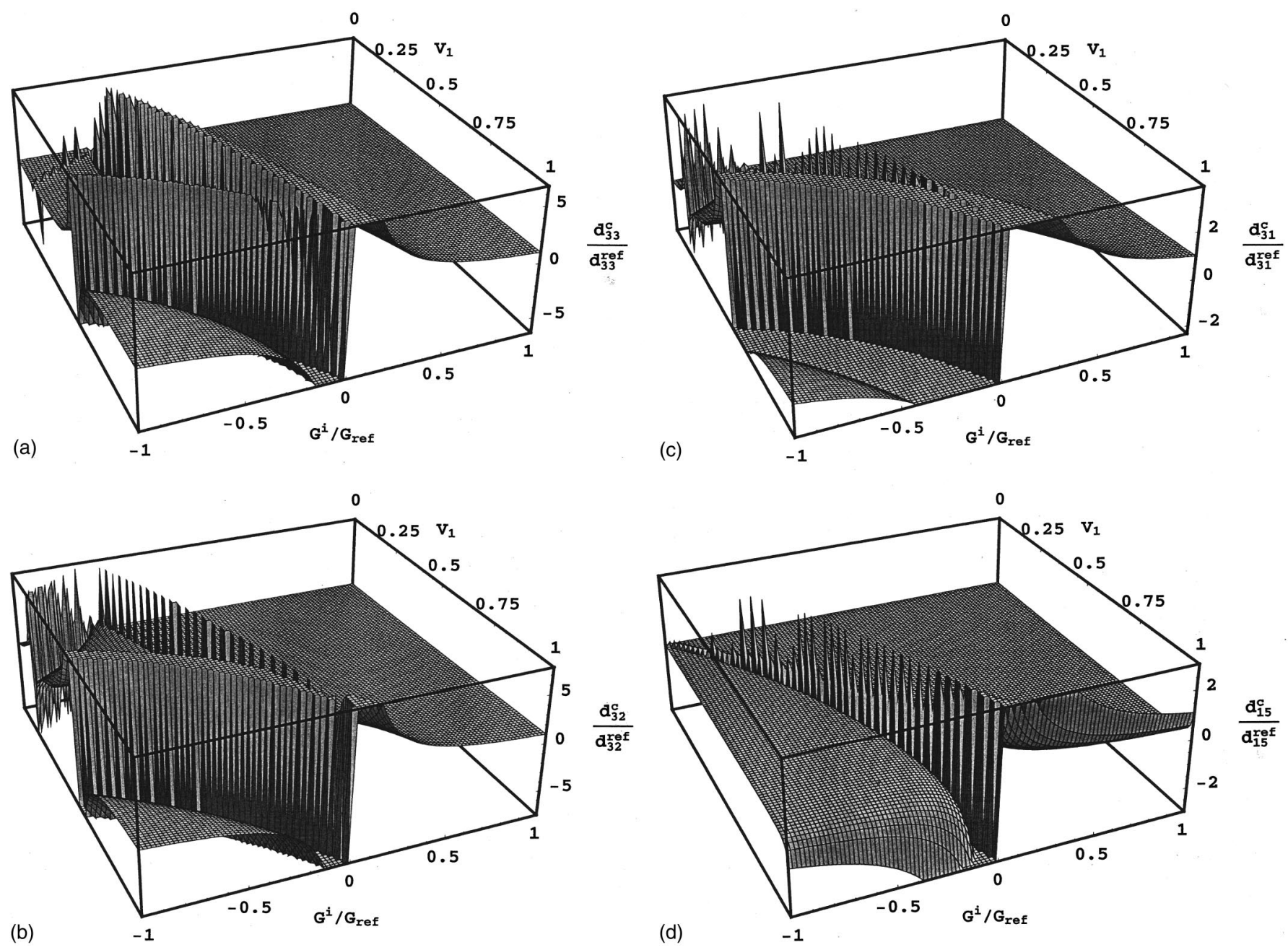

FIG. 7. (a) Normalized piezoelectric longitudinal coefficient $d_{33}$, (b) normalized piezoelectric transverse coefficient $d_{32}$, (c) normalized piezoelectric transverse coefficient $d_{31}$, and (d) normalized piezoelectric shear coefficient $d_{15}$ of a 1-3 fiber-type piezoelectric composite versus inclusion volume fraction $\left(V_{1}\right)$ and normalized inclusion shear modulus $\left(G^{i} / G_{\text {ref }}\right)$, where $G_{\text {ref }}=25 \mathrm{GPa}$. Calculated by Eqs. (14)-(24).

be increased by using composites. ${ }^{36}$ In the present work, it has been shown that piezoelectric and other coupled field properties can become unbounded, not just increased.

To extend our idea to predict other anomalies in other coupled fields, phenomenologically, the effective moduli of two-phase composites with coupled multiple fields, such as

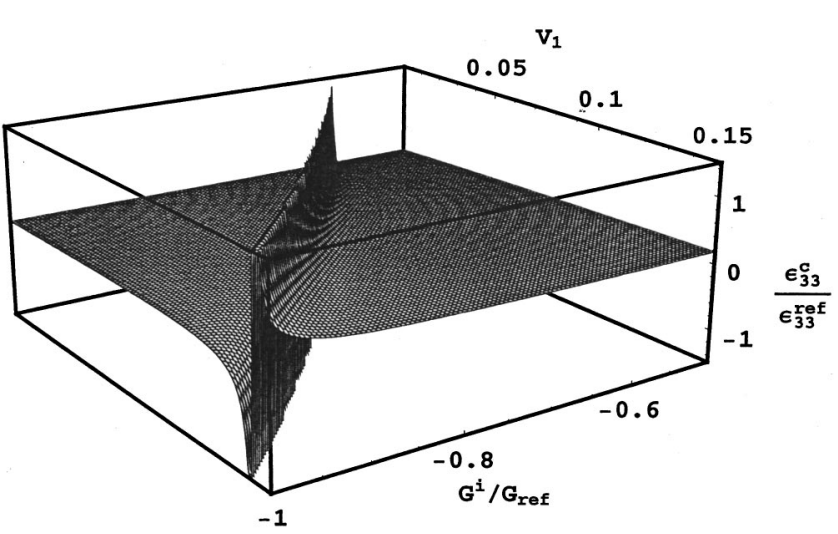

FIG. 8. Normalized permittivity constant $\epsilon_{33}$ of a 1-3 fiber-type piezoelectric composite versus inclusion volume fraction $\left(V_{1}\right)$ and normalized inclusion shear modulus $\left(G^{i} / G_{\text {ref }}\right)$, where $G_{\text {ref }}=25$ GPa. Calculated by Eq. (23). thermoelectric, magnetoelectric, and piezoelectric effects, can be obtained exactly by a modulus matrix decomposition approach. ${ }^{37}$ Therefore, the overall properties can be exactly calculated simply by summing a finite series, whose terms

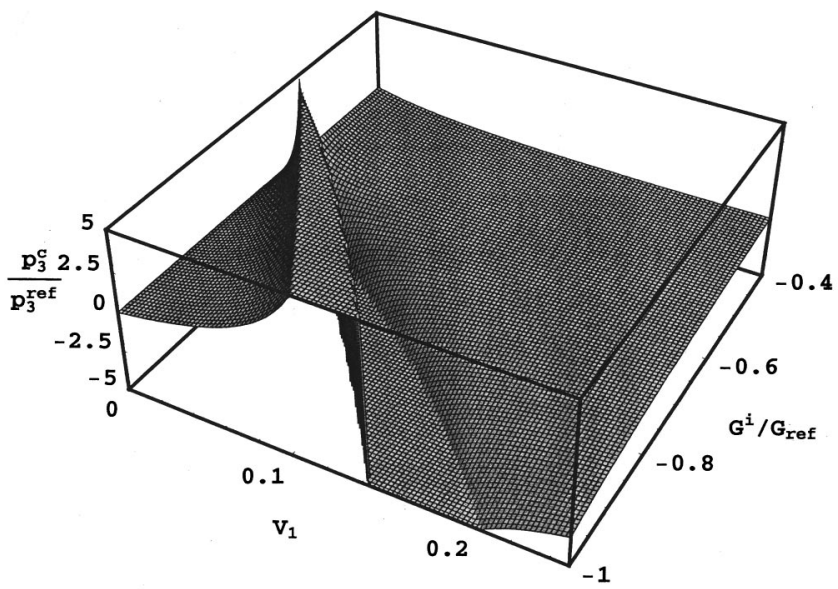

FIG. 9. Normalized pyroelectric coefficient $p_{3}$ of a 1-3 fiber-type piezoelectric composite versus inclusion volume fraction $\left(V_{1}\right)$ and normalized inclusion shear modulus $\left(G^{i} / G_{\text {ref }}\right)$, where $G_{\text {ref }}=25 \mathrm{GPa}$. Calculated by Eq. (24). 
are comprised of the phase moduli and volume fractions only. By allowing negative stiffness inclusions, we violate the common assumption of positive definiteness for moduli. In particular, it is possible to exceed commonly accepted bounds. The consequences of negative stiffness inclusions in mechanical systems have been explored theoretically ${ }^{11}$ and experimentally. ${ }^{12,13}$ Therefore, due to negative eigenvalues of the modulus matrix as the coefficients of the terms in the finite series, its sum, which represents the effective moduli, may lead to extremely small or large values of properties, whereas the magnitude of the influence depends on the interaction of the degree of fields. The present theoretical analysis supports this argument. In thermoelastic and piezoelectric materials, elasticity is coupled with temperature and electric field, respectively. Consequently, composites with negative stiffness inclusions may find use in high performance sensors and actuators based on coupled fields.

\section{CONCLUSION}

Extreme coupled field properties including thermal expansion, piezoelectricity, and pyroelectricity can occur in composites in which one phase has negative stiffness. In linearly elastic materials, the properties become singular when positive and negative stiffness are appropriately balanced. The presence of time or frequency dependence in elastic or electric properties, or their coupling will smooth out the singularities to finite peaks. Following the same idea, it also can be envisaged that similar anomalies can be found in other coupled field problems.

\section{ACKNOWLEDGMENT}

The authors are grateful to the NSF for Grant No. CMS9896284.

${ }^{1}$ J. L. Cribb, Nature (London) 220, 576 (1963).

${ }^{2}$ S. Torquato, J. Appl. Phys. 67, 7223 (1990).
${ }^{3}$ R. S. Lakes, J. Mater. Sci. Lett. 15, 475 (1996).

${ }^{4}$ R. S. Lakes, J. Mech. Behav. Mater. 7, 85 (1996).

${ }^{5}$ O. Sigmund and S. Torquato, Appl. Phys. Lett. 69, 3203 (1996).

${ }^{6}$ R. S. Lakes, Science 235, 1038 (1987).

${ }^{7}$ G. Milton, J. Mech. Phys. Solids 40, 1105 (1992).

${ }^{8}$ R. S. Lakes, J. Mater. Sci. 26, 2287 (1991).

${ }^{9}$ T. A. Mary, J. S. O. Evans, T. Vogt, and A. W. Sleight, Science 272, 90 (1996).

${ }^{10}$ R. H. Baughman and D. S. Galvão, Chem. Phys. Lett. 240, 180 (1995).

${ }^{11}$ R. S. Lakes, Phys. Rev. Lett. 86, 2897 (2001).

${ }^{12}$ R. S. Lakes, Philos. Mag. Lett. 81, 95 (2001).

${ }^{13}$ R. S. Lakes, T. Lee, A. Bersie, and Y. C. Wang, Nature (London) 410, 565 (2001).

${ }^{14}$ Z. Hashin, J. Appl. Mech. 50, 481 (1983).

${ }^{15}$ R. A. Schapery, J. Compos. Mater. 2, 380 (1968).

${ }^{16}$ V. M. Levin, Proc. Acad. Sci. of the USSR, Mechanics of solids (MTT) N 6, 137 (1976).

${ }^{17}$ Z. Hashin and S. Shtrikman, J. Mech. Phys. Solids 11, 127 (1963).

${ }^{18} \mathrm{G}$. W. Milton, in Homogenization and effective moduli of materials and media, edited by J. L. Erickson, D. Kinderlehrer, R. Kohn, and J. L. Lions (Springer, Berlin, 1986), pp. 150-175.

${ }^{19}$ D. P. Skinner, R. E. Newnham, and L. E. Cross, Mater. Res. Bull. 13, 599 (1978).

${ }^{20}$ J. F. Nye, Physical properties of crystals: their representation by tensors and matrices (Clarendon, Oxford, 1957).

${ }^{21}$ Y. Benceniste and G. J. Dvorak, J. Mech. Phys. Solids 40, 1295 (1992).

${ }^{22}$ J. Li and M. L. Dunn, J. Appl. Phys. 89, 2893 (2001).

${ }^{23}$ Y. Benveniste, Mech. Mater. 18, 183 (1994).

${ }^{24}$ K. Schulgasser, J. Mech. Phys. Solids 40, 473 (1992).

${ }^{25}$ M. Milgrom and S. Shtrikman, Phys. Rev. A 40, 1568 (1989).

${ }^{26}$ R. M. Christensen and K. H. Lo, J. Mech. Phys. Solids 27, 315 (1979).

${ }^{27}$ C. W. Nan and F.-S. Jin, Phys. Rev. B 48, 8578 (1993).

${ }^{28}$ C.-W. Nan, Phys. Rev. B 49, 12619 (1994).

${ }^{29}$ R. Hill, J. Mech. Phys. Solids 13, 213 (1963).

${ }^{30}$ R. Hill, J. Math. Phys. 5, 548 (1964).

${ }^{31}$ J. Li and G. J. Weng, Proc. R. Soc. London, Ser. A 455, 3493 (1999).

${ }^{32}$ M. Tabib-Azar, Microactuators (Kluwer, Dordrecht, 1998).

${ }^{33}$ R. S. Lakes and W. Drugan (unpublished).

${ }^{34}$ N. A. Nicorovici, R. C. Mcphedran, and G. W. Milton, Phys. Rev. B 49, 8479 (1994)

${ }^{35}$ W. A. Smith and B. A. Auld, IEEE Trans. Ultrason. Ferroelectr. Freq. Control 38, 40 (1991)

${ }^{36}$ C. Cui, R. Baughman, Z. Iqbal, T. R. Kazmar, and D. K. Dahlstrom, Synthetic Metals 85, 1391 (1997).

${ }^{37}$ T. Chen, J. Mech. Phys. Solids 45, 385 (1997). 